\title{
Sostener el discurso científico en aulas de 4 y 5 años: análisis de las intervenciones de docentes y alumnado y del conocimiento emergente en torno al huerto ecológico escolar
}

\author{
Lourdes Aragón $^{1}$ \\ Susana Sánchez ${ }^{1}$ C \\ Vanesa García Salado ${ }^{1}$ \\ ${ }^{1}$ Universidad de Cádiz (UCA), España
}

Resumen. Este trabajo estudia el discurso compartido en aulas de Educación Infantil como parte del proceso de alfabetización científica. Se analizan las intervenciones de docentes y niños de 4 y 5 años en el discurso compartido y los esquemas de conocimiento que emergen durante asambleas en torno al huerto ecológico escolar. Las intervenciones han sido transcritas y analizadas con un sistema emergente de categorías consensuado entre expertos. Los resultados indican que la intervención docente en 4 y 5 años se orienta principalmente a la petición de información y aceptación por repetición. Se observa una mayor frecuencia en 5 años de aceptación por reelaboración y retar planteamientos. Respecto de la participación infantil, tanto el grupo de 4 años como el de 5 repiten, elaboran y reestructuran la información dada, si bien en 5 años es menos frecuente la simple repetición. En cuanto al avance conceptual, se identifican respectivamente para las aulas de 4 y 5 años un total de 30 y 56 esquemas de conocimiento vinculados a modelos iniciales del ciclo de vida de las plantas y la idea infantil de huerto. Se concluye que el huerto ecológico es un contexto adecuado para propiciar el discurso científico en edades tempranas.

Palabras clave: asamblea de aula, competencia científica, educación infantil, huerto ecológico escolar, discurso científico, esquemas mentales.

Sostener el discurso científico en aulas de 4 y 5 años: análisis de las intervenciones de docentes y alumnado y del conocimiento emergente en torno al huerto ecológico escolar Resumo. Este trabalho estuda o discurso compartilhado em salas de aula de Educação Infantil como parte do processo de alfabetização científica. São analisadas as intervenções de professores e crianças de 4 e 5 anos no discurso compartilhado e os esquemas de conhecimento que surgem durante as assembleias em torno da horta ecológica escolar. As intervenções foram transcritas e analisadas com um sistema de categorias emergentes acordado entre os especialistas. Os resultados indicam que a intervenção pedagógica em crianças de 4 e 5 anos está principalmente orientada a solicitação de informação e aceitação por repetição. Uma maior frequência é observada em crianças de 5 anos de idade de aceitação, através de abordagens de retrabalho e desafio. Em relação à participação infantil, tanto o grupo de 4 anos como o de 5 anos repetem, elaboram e reestruturam as informações fornecidas, embora em crianças de 5 anos a repetição simples seja menos frequente. Em relação ao avanço conceitual, são identificados um total de 30 e 56 esquemas de conhecimento ligados a modelos iniciais do ciclo de vida das plantas e à ideia infantil de jardim, respetivamente para as salas de 4 e 5 anos. Concluímos que a horta ecológica é um contexto adequado para promover o discurso científico desde cedo.

Palavras-chave: montagem de sala de aula, concurso científico, educação infantil, horta escolar ecológica, discurso científico, esquemas mentais.

Sustaining scientific discourse in 4- and 5-year-old classrooms: analysis of teachers' and students' interventions and emerging knowledge about the school ecological garden Abstract. This work studies the shared discourse in Early Childhood Education classrooms as part of the scientific literacy process. The interventions of teachers and children aged 4 and 5 are analysed in the shared discourse and knowledge schemas that emerge during assemblies around the school ecological garden. The interventions have been transcribed and analysed with an emergent system of categories agreed among experts. The results indicate that the teaching intervention in 4 and 5 year old is mainly oriented towards information request and acceptance by repetition. A higher frequency is observed in 5 years of approval by reworking and challenging. Regarding children's participation, both 4 and 5 year old repeat, elaborate and restructure the information given, although in 5 year olds simple repetition is less frequent. Furthermore, looking at the conceptual progress, a total of 30 and 56 knowledge schemas linked to initial models of the life cycle of plants and the children's idea of a vegetable garden were identified for the 4 and 5 year old classrooms, respectively. Finally, it can be concluded that the organic garden is a suitable context for promoting scientific discourse at an early age. Keywords: Circle time, scientific competence, early childhood education, school organic garden, scientific classroom, discourse, mental schemas. 


\section{Introducción}

La escuela actual busca un cambio didáctico profundo, que plantee metodologías de enseñanza más participativas y activas, para el desarrollo de habilidades científicas y un pensamiento crítico (Garzón y Martínez, 2017). En Educación Infantil (EI), algunos estudios evidencian una escasa presencia de actividades claves para el aprendizaje de las ciencias en las aulas (Cantó et al. 2016). Según García-Carmona et al. (2014) la regulación española de enseñanzas mínimas para 3-6 años (R.D. 1630/2006, de 29 de diciembre) contempla, de manera explícita y adecuada, el desarrollo de las capacidades propias de la indagación científica y de otras competencias básicas esenciales en la educación científica como la comunicativa, matemática, cultural y digital. Sin embargo, estos autores detectan carencias en el currículum en lo que respecta a la adquisición de competencias relacionadas con la socialización y la afectividad, esenciales para promover una adecuada alfabetización científica desde etapas iniciales.

Por otra parte, la competencia científica, tanto personal como social, incluye "el conjunto integrado de capacidades para utilizar el conocimiento científico a fin de describir, explicar y predecir fenómenos naturales para comprender los rasgos característicos de la ciencia, para formular e investigar problemas e hipótesis, así como para argumentar y tomar decisiones personales y sociales sobre el mundo natural y los cambios que la actividad humana genera en él" (Mérida et al., 2017, p.20). Ese proceso de construcción del conocimiento científico escolar, al igual que ocurre en ciencia con el lenguaje que se genera en las discusiones entre científicos, por ejemplo, es considerado como una de las variables fundamentales (Sanmartí, 2002). Sin embargo, de acuerdo con Izquierdo y Sanmartí (1999), cuando se habla de las habilidades que deben ser enseñadas para aprender ciencias de la naturaleza, pocas veces se priorizan aquellas más relacionadas con la expresión y la comunicación de las ideas, es decir, describir, definir, explicar o argumentar, frente a otras a las que, quizás, se les otorga más importancia, como observar, plantear hipótesis o diseñar experimentos. Es por ello que en las aulas podría darse más importancia a la discusión de las ideas y al uso de un lenguaje personal sobre el que habituarse a argumentar, como paso previo para que el alumnado comience a apropiarse del lenguaje de la ciencia (Sardá y Sanmartí, 2000).

Iniciar el proceso de alfabetización científica en edades tempranas requiere de docentes capaces de diseñar propuestas bien planificadas en el aula que integren procesos científicos (De la Blanca et al., 2013) sobre los que se construyan modelos iniciales, precursores de modelos científicos escolares más complejos que serán trabajados en etapas sucesivas (Canedo-Ibarra et al., 2012; Mazas et al., 2018). Además, siguiendo a García-Carmona et al. (2014) ante el currículum de infantil, es necesario emplear el medio como escenario para el aprendizaje de las ciencias, entendiendo este en su sentido más amplio y utilizando una amplia diversidad de recursos didácticos, no solo dentro del aula, también fuera de ella. En este sentido, el huerto toma un papel especial. Estudios recientes evidencian cómo el huerto ecológico puede ser empleado como contexto de enseñanza-aprendizaje de las ciencias de la naturaleza, no solo a la hora de trabajar conceptos vinculados a contenidos propios de esta área 
(anatomía y diversidad de las plantas, gestión del agua como recurso), también por desarrollar procedimientos científicos y actitudes positivas hacia el entorno más cercano (Eugenio-Gozalbo, et al., 2020).

Desde esta perspectiva, y a tenor de los resultados obtenidos en investigaciones recientes (Aragón et al., 2021), el presente trabajo tiene como principal objetivo profundizar en el recurso del huerto ecológico y su presencia en los momentos de asamblea. Se pretende analizar de qué forma estos espacios de aprendizaje permiten aflorar esquemas de conocimientos en niños de 4 y 5 años y qué estrategias ponen en juego los docentes para gestionar ese discurso científico compartido con el grupo para propiciar el avance de la competencia científica del alumnado.

\section{Marco teórico}

Existe una larga tradición de estudios sobre la construcción compartida del discurso en el aula y su relación con el aprendizaje efectivo (Mercer et al., 2019) que muestra un avance desde un habla dominante por parte del docente (preguntas cerradas al alumnado y evaluación posterior) que se ha ido sustituyendo por modalidades de interacción más flexibles y adaptadas a una concepción de aprendizaje significativo, en el que la actividad de enseñanza y aprendizaje incluye la explicitación de conocimientos previos del alumnado y se basa en procesos dialógicos de construcción del conocimiento (Wells, 2001; Baker et al., 2019). Esto implica un uso elevado y consciente del lenguaje, con matices y profundidad, que no subestime las capacidades infantiles, que integre sus ideas y con ello asuma de forma decidida el desarrollo de una alfabetización epistémica (Wells, 1987; Mercer, 2017) en la infancia vinculado al avance en la competencia científica del alumnado (Sardá y Sanmartí, 2000). Consideramos, en línea con Monteira y Jiménez-Aleixandre (2016; 2019) que este compromiso es posible desde edades tempranas, en paralelo al acceso infantil a la oralidad formal y la lengua escrita.

El espacio de la asamblea, consolidado en la escuela infantil como espacio de comunicación oral por excelencia puede permitir el desarrollo de los aspectos más comunicativos de la competencia científica desde una gestión adecuada de la comunicación en el grupo (Díez, 1998; Pozuelos, 2007; Mérida et al., 2015; Sánchez, 2016; Sánchez y González, 2016) e incluir en ella aspectos afectivos y de socialización, como demandan García-Carmona et al. (2014). Los estudios indican que la calidad de gestión del docente en estos espacios de comunicación compartida es determinante para la efectividad de los enfoques dialógicos del aprendizaje de las ciencias (Wells, 2001; Mortimer y Scott, 2003; Warwick y Cook, 2019).

En relación con el uso del huerto escolar, ampliamente extendido en España (Germinando, 2018), diversos estudios evidencian su enorme potencial didáctico: supone romper con un modelo teórico de enseñanza, aproximar al alumnado a un espacio abierto y vincular el medio con los problemas que se pueden encontrar en él (Marques y Cuéllar, 2021; Armienta et al., 2019).

El huerto escolar permite mejorar el conocimiento de los estudiantes sobre la producción de alimentos, estimula la creatividad en la búsqueda compartida de soluciones a problemas que se generan en torno a este espacio (Marques y Cuéllar, 2021); mejora significativamente habilidades científicas como la toma de datos y 
medidas, promoviendo una actitud favorable hacia las ciencias, o la adquisición de hábitos saludables (Carballido et al., 2021). También permite desarrollar conceptos estructurantes como el de ecosistema y el pensamiento sistémico (Sáez et al., 2017), ambos fundamentales para poder entender y actuar ante muchos de los problemas socioambientales actuales (García, 2003). Rodríguez-Marín et al. (2015) inciden en el interés del huerto para el desarrollo de la conciencia ambiental del alumnado frente a la situación de emergencia climática desde marcos alternativos como el decrecimiento. El huerto, así, permite integrar modelos didácticos basados en la investigación escolar en aulas de niños y niñas de 3 años, iniciándose así en la alfabetización ambiental a través del contacto con la naturaleza (Rodríguez-Marín et al., 2021). Sin embargo, aún existe un amplio margen de mejora con relación al uso del huerto para fomentar habilidades propias de la ciencia en El (Alcántara et al., 2019), por ello, consideramos relevante seguir profundizando en estudios como el que presentamos, que evidencien el uso del huerto ecológico escolar para favorecer el desarrollo de la competencia científica desde edades tempranas y según propuestas actuales (Eugenio y Aragón, 2016).

\section{Cuestiones de investigación}

Nos proponemos analizar el funcionamiento del discurso compartido generado en asamblea de aula y en el marco de un proyecto de diseño y puesta en marcha de un huerto ecológico en la El. El objetivo general es valorar si el huerto es un contexto de aprendizaje adecuado para promover el discurso científico en la EI. Para ello, este estudio se centra: 1) en las intervenciones de los docentes y del alumnado en el discurso compartido y 2) en los esquemas de conocimiento que emergen en dicho discurso. Para dar respuesta a nuestro objetivo general se plantean las siguientes cuestiones de investigación:

- ¿Cómo gestionan los docentes el discurso científico en distintas sesiones de asamblea en un aula de 4 años y otra de 5 ?

- ¿Cómo participa el alumnado en el discurso científico compartido?

- ¿Qué esquemas de conocimiento afloran en dichas sesiones?

\section{Metodología}

\subsection{Enfoque metodológico}

Partimos de un enfoque metodológico cualitativo enmarcado en un paradigma interpretativo, pues se pretende estudiar los significados de las acciones humanas para así interpretar situaciones que afectan a un contexto educativo (Latorre et al., 2003). Se encuentra próximo a un estudio de casos de tipo múltiple (Stake, 2005) en el que se abordan dos aulas, cada una correspondiente a una subunidad de análisis, según Yin (2009). Ambas aulas pertenecen a un mismo centro educativo y la actividad desarrollada en ellas comparte un mismo contexto y planteamiento. 


\subsection{Escenario del contexto y participantes}

La investigación se enmarca en un proyecto de investigación más amplio financiado por la Junta de Andalucía (PIV-040/17) dirigido a evaluar el uso del huerto ecológico como contexto de aprendizaje para el desarrollo de la competencia científica en El. En este trabajo nos centramos en dos aulas, ambas con 25 niños, que pertenecen al CEIP Reyes Católicos de Cádiz (España):

- Aula de 4 años: 15 niños y 10 niñas y su tutora (26 años de experiencia).

- Aula de 5 años: 15 niños y 10 niñas y su tutor (16 años de experiencia).

Se diseñó e implementó una secuencia didáctica en torno al huerto, en el que se alternaban actividades individuales, en pequeño grupo y en gran grupo. En Aragón et al. (2021) se expone en detalle la propuesta diseñada.

\subsection{Instrumentos de recogida de información}

Se realizaron una serie de reuniones entre investigadoras y docentes para consensuar el sentido de las asambleas que la secuencia didáctica incluía, así como para orientar su gestión del discurso compartido con el alumnado. Se realizaron tres y cuatro asambleas respectivamente por aula, que fueron grabadas en audio y vídeo por las investigadoras, y posteriormente transcritas. Algunos fragmentos ajenos al discurso ligado a la secuencia fueron eliminados.

Se trazaron diferentes pautas de intervención docente para las asambleas, de forma que su organización secuencial permitiera (1) acceder a los conocimientos o esquemas previos del alumnado; (2) profundizar en la estructura de dichos conocimientos; (3) consolidar un conocimiento basado en la evidencia de la experiencia de la puesta en marcha del huerto ecológico.

Para este trabajo tomamos en consideración las tres asambleas que se realizaron paralelamente en ambos cursos de 4 y 5 años, para establecer una comparación entre ellas en lo que respecta a la gestión de la comunicación por parte del docente y a la participación del alumnado en la construcción conceptual del discurso. En la Tabla 1 se expone el sentido de las tres asambleas analizadas.

Tabla 1. Descripción de las asambleas desarrolladas.

\begin{tabular}{|c|c|c|}
\hline Asamblea & Objetivo & Pautas en la intervención docente \\
\hline Inicial & $\begin{array}{l}\text { Explorar esquemas mentales previos } \\
\text { del alumnado sobre temas relaciona- } \\
\text { dos con el huerto ecológico. }\end{array}$ & $\begin{array}{l}\text { Gestión dirigida a compartir el conoci- } \\
\text { miento: foco en el contenido expresado } \\
\text { sobre la exigencia de participación. }\end{array}$ \\
\hline Segunda & $\begin{array}{l}\text { Profundizar en el conocimiento pre- } \\
\text { vio y puesta en común para su avan- } \\
\text { ce mediante la elaboración de una } \\
\text { lista de elementos necesarios en un } \\
\text { huerto por orden de importancia. }\end{array}$ & $\begin{array}{l}\text { Gestión dirigida a promover la expre- } \\
\text { sión y justificación de relaciones entre } \\
\text { elementos del huerto. }\end{array}$ \\
\hline Tercera & $\begin{array}{l}\text { Discutir y argumentar sobre los fenó- } \\
\text { menos observados en cuatro experi- } \\
\text { mentos sobre las plantas. }\end{array}$ & $\begin{array}{l}\text { Gestión dirigida promover la expresión } \\
\text { de un conocimiento consolidado basa- } \\
\text { do en la evidencia científica }\end{array}$ \\
\hline
\end{tabular}




\subsection{Análisis de datos}

Las asambleas fueron analizadas desde una perspectiva etnográfica: se ha considerado el discurso construido en interacción como un producto colectivo (Mercer, 2017; Monteira y Jiménez-Aleixandre, 2019), teniendo en cuenta la distinta posición que adquieren docente y alumnado en dicho discurso, y se ha considerado que pueda haber intervenciones que adquieran más de una función simultáneamente (Leifstein y Snell, 2011; 2019). Esto nos ha llevado a explorar nuestros datos y elaborar un sistema de categorías emergentes que permitan comprender el funcionamiento del discurso analizado. Dichas categorías se identificaron tras el análisis de los datos del aula de 5 años y se aplicaron posteriormente al aula de 4 años.

Tras dividir las transcripciones en secuencias, definidas por su coherencia semántica, se procedió a analizar los datos en dos niveles, que se corresponden con lo que Lemke (1997) denominó respectivamente patrón estructural y patrón temático. El primer nivel consistió en estudiar los procesos de construcción conjunta del discurso científico, esto es, los turnos de intervención de alumnado y docente. El segundo nivel abordó la organización conceptual presente en el discurso compartido, es decir, explorar qué esquemas de conocimiento científico afloraron en las asambleas a propósito del huerto ecológico. Estos esquemas han podido ser aportados por docente o alumnado, y se contemplan desde una perspectiva de conocimiento en construcción.

\subsubsection{Análisis del primer nivel: intervenciones de alumnado y docente}

Para las intervenciones de los docentes, se identificaron las categorías emergentes que figuran en la tabla 2 (Aragón y Sánchez, aceptado).

Tabla 2. Sistema de categorías emergentes de análisis del discurso del docente.

\begin{tabular}{|c|c|c|}
\hline $\begin{array}{l}\text { Categoría discurso } \\
\text { docente }\end{array}$ & Descripción & Ejemplos (N: niño/a y M: Docente) \\
\hline 1. Petición & $\begin{array}{l}\text { Demanda información del } \\
\text { alumnado }\end{array}$ & $\begin{array}{l}\text { "M: a ver por ahí ¿qué pensáis, ¿qué ha } \\
\text { salido o que no la semilla?" }\end{array}$ \\
\hline 2. Acepta (repetición) & $\begin{array}{l}\text { Acepta la información dada } \\
\text { por el alumnado repitiéndola } \\
\text { textualmente }\end{array}$ & $\begin{array}{l}\text { "N: no va a sobrevivir; M: no va a so- } \\
\text { brevivir" }\end{array}$ \\
\hline $\begin{array}{l}\text { 3. Acepta (reelabora- } \\
\text { ción) }\end{array}$ & $\begin{array}{l}\text { Acepta la información dada } \\
\text { por el alumnado reelaboran- } \\
\text { do el discurso para mayor } \\
\text { precisión }\end{array}$ & $\begin{array}{l}\text { "N: que que todavía están creciendo, } \\
\text { pero están en la tierra", M: claro están } \\
\text { creciendo, pero está debajo en la tierra, } \\
\text { es que todavía no ha llegado arriba tar- } \\
\text { dan más en salir porque como estaba } \\
\text { aquí abajo..." }\end{array}$ \\
\hline 4. Se opone o rectifica & $\begin{array}{l}\text { No acepta la información } \\
\text { dada por los niños }\end{array}$ & $\begin{array}{l}\text { "N: los cactus parecen como plantas, } \\
\text { pero no tienen hojas tiene pinchos, pero } \\
\text { si lo tocas parece que pincha" M: pero } \\
\text { los cactus no son plantas carnívoras eso } \\
\text { son otro tipo de plantas, hay muchos } \\
\text { tipos de plantas". }\end{array}$ \\
\hline
\end{tabular}




\begin{tabular}{ccc}
\hline $\begin{array}{c}\text { Categoría discurso } \\
\text { docente }\end{array}$ & \multicolumn{1}{c}{ Descripción } & \multicolumn{1}{c}{ Ejemplos (N: niño/a y M: Docente) } \\
5. Apoya continuidad & Da pie a que quien habla "N: sí, pero las plantas hay muchos tipos \\
& continúe su discurso & de plantas. \\
& & M: ¿y tú crees que todas necesitan agua \\
& o solo algunas? \\
& N:algunasy otras pueden, otras si quieren \\
& echarle agua le echan agua si no quieren \\
pues no la echan".
\end{tabular}
complementaria formación más grande, ya han germinado mucho y habría que trasplantarlas".

7. Explicita posiciones Hace explícita información "M: tú piensas que semillas...y tú que que ha sido construida en el agua..." discurso

8. Confronta posiciones Presenta informaciones con- "M: a ver, dice él que el sol no se planta trastantesque han sidocons- $y$ yo le he preguntado ¿hace falta el sol truidas en el discurso para un huerto? $Y$ ella dice que sí hace falta ¿por qué has dicho sol?, ¿por qué crees tú que hace falta el sol?, ¿qué hacemos?, ¿qué ponemos?"

9. Reta planteamientos Exige repensarlainformación "M: ¿y por qué pensáis que en el algoque están manejando dón sale la semilla si el algodón no es la tierra?, ¿por qué será?"

10. Promueve acuerdo Propone acordar posiciones "M: pues entonces ponemos que hay queso en el huerto, y vamos a poner también los garbanzos del huerto en el listado".

11. Explicita acuerdo Expone la información refren- "M: ya hemos dicho que el agua, ya hemos dada por el acuerdo pensado todosque sí, que nos hacía falta, pero ahora otra cosa que decíamos que nos hacía falta era la tierra"

12. Valora, felicita, anima Motiva y valora la partici- "M: regar... muy bien!" pación

13. Proyecta actividad Plantea próxima actividad "M: Bueno pues esto que decís del algofutura. posible dón que aquí crece igual, vamos a hacer una cosa, vamos a seguir viéndolas y vamos a ver estas plantas las que están en el algodón cuando se hagan grandes si siguen aquí en el algodón, y las que tenemos en tierra eh, vale, ok?"

Fuente: elaboración propia.

En lo que respecta al alumnado, se estudió en primer lugar de qué forma sus intervenciones contribuían al avance del discurso compartido, y, en segundo lugar, se profundizó en el sentido conceptual de dichas intervenciones, considerando si suponían aporte informativo, aporte de esquemas de conocimiento, argumentación o modelización de esquemas (Aragón et al., 2021). Para ambos análisis se identificaron categorías emergentes. En este estudio nos centramos en cómo las intervenciones infantiles contribuyen al avance del discurso compartido, según el sistema de categorías que presentamos en la Tabla 3. 
Tabla 3. Sistema de categorías emergentes de análisis del discurso del alumnado.

\begin{tabular}{|c|c|c|}
\hline $\begin{array}{l}\text { Categoría discurso } \\
\text { alumnado }\end{array}$ & Descripción & $\begin{array}{c}\text { Ejemplos } \\
\text { (N: niño/a y M: Docente) }\end{array}$ \\
\hline 1. Da información & Interviene en el discurso & $\begin{array}{l}\text { "M. ¿qué es un huerto? } \\
\mathrm{N} \text { : Donde hay tomateras" }\end{array}$ \\
\hline 2. Repite información & $\begin{array}{l}\text { Interviene con información } \\
\text { ya aportada previamente al } \\
\text { discurso }\end{array}$ & $\begin{array}{l}\text { "[Se ha dicho previamente que un huerto } \\
\text { es donde hay verduras] } \\
\text { M: ¿Donde hay? } \\
\mathrm{N} \text { : verduras" }\end{array}$ \\
\hline $\begin{array}{l}\text { 3. Elabora informa- } \\
\text { ción }\end{array}$ & $\begin{array}{l}\text { Avanza en información ya } \\
\text { aportada }\end{array}$ & "N: donde crecen las verduras" \\
\hline $\begin{array}{l}\text { 4. Reestructura infor- } \\
\text { mación }\end{array}$ & $\begin{array}{l}\text { Replantea información ya } \\
\text { aportada }\end{array}$ & $\begin{array}{l}\text { "M: (..)¿solo crece con el sol la planta? } \\
\mathrm{N} \text { : ¡nooo! necesita también muchísima } \\
\text { agua, pero que no se pase, porque si no } \\
\text { se ahogan... }\end{array}$ \\
\hline 5. Hace pregunta & $\begin{array}{l}\text { Pregunta sobre información } \\
\text { presente en el discurso }\end{array}$ & $\begin{array}{l}\text { "(en relación con la germinación) } \\
\mathrm{N} \text { : que, que que... ¿al nacer nunca se le } \\
\text { tiene que dar prisa? }\end{array}$ \\
\hline 6. Concluye & $\begin{array}{l}\text { Retoma información construi- } \\
\text { da en el discurso dándola por } \\
\text { definitiva }\end{array}$ & $\begin{array}{l}\text { "N: ahora lo sé porque por el sol y por la } \\
\text { lluvia pues la planta va creciendo" }\end{array}$ \\
\hline $\begin{array}{l}\text { 7. Demanda cambio de } \\
\text { tema/actividad }\end{array}$ & $\begin{array}{l}\text { Solicita abordar otro tema o } \\
\text { actividad }\end{array}$ & “N. ¿vamos a ver cómo está el huerto? \\
\hline $\begin{array}{l}\text { 8. Solicita o rechaza } \\
\text { participar }\end{array}$ & $\begin{array}{l}\text { Pide la palabra o rechaza } \\
\text { participar }\end{array}$ & $\begin{array}{l}\text { [Levantan manos o se mantienen en } \\
\text { silencio tras haber sido invitados a hablar] }\end{array}$ \\
\hline $\begin{array}{l}\text { 9. El alumno toma un } \\
\text { rol propio del docente }\end{array}$ & $\begin{array}{l}\text { Se han agrupado los casos } \\
\text { en que el alumnado adopta } \\
\text { un rol docente (demanda } \\
\text { información de algún otro } \\
\text { alumno, por ejemplo). }\end{array}$ & $\begin{array}{l}\text { "N: (a otro niño) pero ¿tú crees que un } \\
\text { bicho es capaz de comer una planta así?" }\end{array}$ \\
\hline
\end{tabular}

Fuente: elaboración propia.

Con objeto de comparar cómo intervienen los docentes y cómo participa el alumnado por grupo-clase y asamblea, se utilizó la prueba $Z$ de diferencia de proporciones. Para ello, se estableció como valor crítico $Z=1,96$ para la prueba de una sola cola y un nivel de confianza del $95 \%$.

\section{Resultados}

5.1 ¿Cómo gestionan los docentes el discurso científico en distintas sesiones de asamblea un aula de 4 años y otra de 5?

Al comparar las intervenciones docentes para cada una de las asambleas y aula, los resultados muestran diferencias estadísticamente significativas para algunas de las categorías establecidas (Tabla 4). 
Tabla 4. Análisis comparativo del porcentaje de intervenciones de los docentes de las aulas de 4 y 5 años por asambleas ( $N=n^{0}$ de intervenciones totales).

\begin{tabular}{|c|c|c|c|}
\hline \multicolumn{4}{|c|}{ Asamblea 1} \\
\hline & 4 años & 5 años & \\
\hline Categorías emergentes de análisis del discurso & $N=163$ & $\mathrm{~N}=201$ & z \\
\hline 1. Petición & 31,3 & 23,9 & 1,58 \\
\hline 2. Acepta por repetición & 22,1 & 21,9 & 0,04 \\
\hline 3. Acepta reelaborando & 6,7 & $15,4^{*}$ & $-2,58$ \\
\hline 4. Se opone o rectifica la intervención & 2,5 & 1,0 & 1,09 \\
\hline 5. Apoya continuidad del discurso & 29,4 & 23,9 & 1,20 \\
\hline 6. Aporta información complementaria & 3,7 & $0,0 *$ & 2,74 \\
\hline 7. Explicita posiciones de los niños & 0 & $5,5 *$ & $-3,03$ \\
\hline 8. Confronta posiciones de los niños & 0 & 1,0 & $-1,28$ \\
\hline 9. Reta planteamientos niños & 0 & $5,0 *$ & $-2,89$ \\
\hline 10. Promueve acuerdo & 0 & 0,0 & 0,00 \\
\hline 11. Explicita acuerdo & 0 & 0,0 & 0,00 \\
\hline 12. Anima, felicita, valora & 3,1 & 1,5 & 1,02 \\
\hline 13. Proyecta actividad futura & 1,2 & 1,0 & 0,21 \\
\hline \multicolumn{4}{|c|}{ Asamblea 2} \\
\hline & 4 años & 5 años & \\
\hline Categorías emergentes de análisis del discurso & $\mathrm{N}=247$ & $N=309$ & Z \\
\hline 1. Petición & 32,0 & $18,1 *$ & 3,79 \\
\hline 2. Acepta por repetición & 23,5 & 22,3 & 0,32 \\
\hline 3. Acepta reelaborando & 2,4 & $14,9 *$ & $-5,01$ \\
\hline 4. Se opone o rectifica la intervención & 4,0 & 5,8 & $-0,95$ \\
\hline 5. Apoya continuidad del discurso & 27,9 & $16,2 *$ & 3,36 \\
\hline 6. Aporta información complementaria & 1,2 & 1,3 & $-0,08$ \\
\hline 7. Explicita posiciones de los niños & 3,6 & 5,5 & $-1,03$ \\
\hline 8. Confronta posiciones de los niños & 2,8 & 1,0 & 1,64 \\
\hline 9. Reta planteamientos niños & 0,8 & $11,3^{*}$ & $-4,94$ \\
\hline 10. Promueve acuerdo & 1,2 & 0,3 & 1,24 \\
\hline 11. Explicita acuerdo & 0 & 1,0 & $-1,55$ \\
\hline 12. Anima, felicita, valora & 0 & $2,3 *$ & $-2,38$ \\
\hline 13. Proyecta actividad futura & 0,4 & 0 & 1,12 \\
\hline
\end{tabular}




\begin{tabular}{lrrr}
\hline \multicolumn{1}{c}{ Asamblea 3 } & & & \\
Categorías emergentes de análisis del discurso & 4 años & 5 años & \\
1. Petición & $\mathrm{N}=209$ & $\mathrm{~N}=282$ & $\mathrm{Z}$ \\
2. Acepta por repetición & 35,4 & $7,4^{*}$ & 7,75 \\
3. Acepta reelaborando & 11,0 & 8,2 & 1,07 \\
4. Se opone o rectifica la intervención & 4,3 & $12,8 *$ & $-3,21$ \\
5. Apoya continuidad del discurso & 2,4 & 4,6 & $-1,29$ \\
6. Aporta información complementaria & 15,8 & $7,8 *$ & 2,77 \\
7. Explicita posiciones de los niños & 19,6 & 16,0 & 1,05 \\
8. Confronta posiciones de los niños & 1,9 & $9,9 *$ & $-3,56$ \\
9. Reta planteamientos niños & 0 & $3,2 *$ & $-2,61$ \\
10. Promueve acuerdo & 5,3 & $21,3 *$ & $-4,99$ \\
11. Explicita acuerdo & 1 & 0,7 & 0,30 \\
12. Anima, felicita, valora & 1,4 & 0,4 & 1,32 \\
13. Proyecta actividad futura & 0,5 & 1,4 & $-1,03$ \\
\hline
\end{tabular}

* Diferencias estadísticamente significativas; Prueba Z de proporciones: Z=1,96; a: 0,05. Fuente: elaboración propia.

En ambas aulas se observa un avance en el discurso que viene condicionado por las funciones que adquieren las asambleas en la secuencia didáctica propuesta. Así, en la asamblea 1, los valores más altos para ambas aulas están en la petición de información, la aceptación por repetición de las aportaciones infantiles y el apoyo a la continuidad del discurso de los niños, como corresponde al objetivo de explorar los conocimientos previos del grupo. Aquello que diferencia a ambas aulas para esta asamblea es la mayor frecuencia de la reelaboración del discurso por parte del tutor de 5 años, y, si bien los valores son bajos, la mayor frecuencia en la que explicita las posiciones de los niños y reta los planteamientos infantiles.

La asamblea 2 coincide en ambas aulas en una alta frecuencia de aceptación por repetición de las intervenciones de los niños, lo cual no es de extrañar al tratarse de una asamblea dirigida a organizar el conocimiento infantil sobre el huerto. Sin embargo, se observan algunas diferencias interesantes: la tutora de 4 años solicita la participación de los niños y debe apoyar la continuidad de su discurso con mucha más frecuencia que en el caso de 5 años, donde se observa una mayor fluidez en este sentido. Esto parece favorecer una mayor exigencia en la intervención con el grupo de 5 años, pues su tutor aumenta significativamente la reelaboración de la información dada por los niños, la frecuencia con la que reta el conocimiento infantil, y quizá como consecuencia de esa mayor exigencia, aparecen -con valores bajos, no obstanteintervenciones dirigidas a animar, felicitar o valorar las aportaciones de los niños.

Finalmente, la asamblea 3, cuyo objetivo era discutir y analizar los fenómenos observados a través de distintos experimentos en torno a elementos del huerto, es la que muestra mayores diferencias entre los dos grupos: si bien los tutores coinci- 
den en aportar información complementaria, lo que indica que aportan más datos y contexto para hacer avanzar los conocimientos infantiles, se dan notables diferencias en la intervención docente. En primer lugar, la maestra de 4 años debe solicitar la intervención de los niños y apoyar la continuidad de su discurso con una frecuencia más elevada que en el caso de 5 años, como ya ocurría en la asamblea 2. En el caso de la petición de intervención, la divergencia aumenta notablemente. También, como ocurría en la asamblea 2, en 5 años es más frecuente la reelaboración de la información por el tutor, y, sobre todo, que rete las aportaciones que hacen los niños. Además, en esta asamblea 3 aparecen otras diferencias entre las dos aulas: el tutor de 5 años explicita o confronta las posiciones infantiles y proyecta nueva actividad sobre la que seguir discutiendo en el futuro. Se observa que la mayor exigencia de discurso para la asamblea 3 resulta más asumible en el aula de 5 años; en el aula de 4 años la tutora, si bien reta las posiciones de los niños en mayor medida que en las asambleas precedentes, debe animar y apoyar la participación para asegurar el avance del discurso. Por tanto, la propuesta didáctica en torno al huerto está siendo efectiva para propiciar un discurso que además de incorporar los conocimientos de los niños, los reta y enriquece con información complementaria que aporta el docente, pero esa mayor exigencia progresiva marca diferencias en la intervención en 4 años -donde se observa una mayor necesidad de sostén por parte de la tutora- y 5 años -donde el tutor se puede permitir ampliar el carácter retador de su discurso-.

Si comparamos las intervenciones de los docentes de ambos cursos en el conjunto de las asambleas (Tabla 5) se observa claramente esta adaptación de los tutores de 4 y 5 años a las necesidades del grupo para lograr el avance de un discurso que aumenta progresivamente de complejidad.

Tabla 5. Análisis comparativo del porcentaje global de intervenciones de los docentes en las aulas de 4 y 5 años. ( $N=n^{0}$ de intervenciones totales).

\begin{tabular}{|c|c|c|c|}
\hline $\begin{array}{c}\text { Categorías emergentes de análisis del } \\
\text { discurso }\end{array}$ & $\begin{array}{c}\text { Docente } \\
4 \text { años (\%) } \\
\text { N=619 }\end{array}$ & $\begin{array}{c}\text { Docente } \\
5 \text { años (\%) } \\
\text { N=792 }\end{array}$ & Z \\
\hline 1. Petición & 33,0 & $15,8^{*}$ & 7,57 \\
\hline 2. Acepta por repetición & 18,9 & 17,2 & 0,84 \\
\hline 3. Acepta reelaborando & 4,2 & $14,3^{*}$ & $-6,30$ \\
\hline 4. Se opone o rectifica la intervención & 3,1 & 4,2 & $-1,09$ \\
\hline 5. Apoya continuidad del discurso & 24,1 & $15,2^{*}$ & 4,30 \\
\hline 6. Aporta información complementaria & 8,1 & 6,2 & 1,38 \\
\hline 7. Explicita posiciones de los niños & 2,1 & $7,1^{*}$ & $-4,30$ \\
\hline 8. Confronta posiciones de los niños & 1,1 & 1,8 & $-0,98$ \\
\hline 9. Reta planteamientos niños & 2,1 & $13,3^{*}$ & $-7,51$ \\
\hline 10. Promueve acuerdo & 0,8 & 0,4 & 1,06 \\
\hline 11. Explicita acuerdo & 0,5 & 0,5 & $-0,05$ \\
\hline 12. Anima, felicita, valora & 1 & 1,8 & $-1,26$ \\
\hline 13. Proyecta actividad futura & 1 & $2,5^{*}$ & $-2,16$ \\
\hline
\end{tabular}

* Diferencias estadísticamente significativa; Prueba Z de proporciones: Z=1,96; a: 0,05

Fuente: elaboración propia. 
Si bien ambos docentes repiten las aportaciones de los niños para aceptarlas con frecuencia similar, adaptan su discurso a la exigencia de cada asamblea: hay un mayor apoyo por parte de la docente de 4 años, que con más frecuencia pide información y apoya la continuidad del discurso del grupo. En cambio, en 5 años, es más frecuente que el maestro rete los planteamientos infantiles, acepte las intervenciones infantiles por reelaboración, explicite las posiciones de los niños y proyecte nueva actividad futura.

\section{2 ¿Cómo participa el alumnado en el discurso científico compartido?}

En el caso de las intervenciones del alumnado en cada una de las asambleas y aula, los resultados muestran también diferencias estadísticamente significativas para algunas de las categorías establecidas (Tabla 6).

Tabla 6. Porcentajes de intervenciones del alumnado por categorías. $\left(\mathrm{N}=\mathrm{n}^{0}\right.$ de intervenciones totales).

\begin{tabular}{lccc}
\hline \multicolumn{4}{c}{ Asamblea 1} \\
4 años & 5 años & \\
Categorías emergentes en el análisis del discurso & $(\mathrm{N}=110)$ & $(\mathrm{N}=156)$ & $\mathrm{Z}$ \\
1. Da información & 8,2 & 14,7 & $-1,62$ \\
2. Repite información & 7,3 & 5,1 & 0,72 \\
3. Elabora información & 54,5 & $35,9^{*}$ & 3,02 \\
4. Reestructura información & 23,6 & 30,8 & $-1,28$ \\
5. Hace pregunta & 0,9 & 0,0 & 1,19 \\
6. Concluye & 0,0 & 3,2 & $-1,90$ \\
7. Demanda cambio de tema/actividad & 0,9 & 0,0 & 1,19 \\
8. Solicita o rechaza participar & 3,6 & 5,1 & $-0,58$ \\
9. El alumno toma un rol propio del docente & 0,9 & 5,1 & $-1,87$ \\
\hline
\end{tabular}

Asamblea 2

Categorías emergentes en el análisis del discurso

4 años $\quad 5$ años

1. Da información

$(\mathrm{N}=193)$

$(\mathrm{N}=237)$

2. Repite información

$8,0 *$

$-2,2$

3. Elabora información

33,7

$5,1^{*}$

4. Reestructura información

29,5

$47,7^{*}$

$-3,8$

5. Hace pregunta

30,1

32,5

$-0,5$

6. Concluye

1,0

0,0

1,6

7. Demanda cambio de tema/actividad

0,0

0,0

0,0

8. Solicita o rechaza participar

0,0

0,0

0,0

9. El alumno toma un rol propio del docente

0,0

$2,1^{*}$

$-2,0$ 


\begin{tabular}{lccc}
\hline \multicolumn{4}{c}{ Asamblea 3} \\
& 4 años & 5 años & \\
Categorías emergentes en el análisis del discurso & $(\mathrm{N}=194)$ & $(\mathrm{N}=225)$ & $\mathrm{Z}$ \\
1. Da información & 4,1 & 5,8 & $-0,77$ \\
2. Repite información & 14,4 & $2,2^{*}$ & 4,63 \\
3. Elabora información & 33,5 & 35,6 & $-0,44$ \\
4. Reestructura información & 33,0 & 40,9 & $-1,67$ \\
5. Hace pregunta & 2,6 & 3,1 & $-0,33$ \\
6. Concluye & 2,1 & 1,8 & 0,21 \\
7. Demanda cambio de tema/actividad & 0,0 & 0,0 & 0,00 \\
8. Solicita o rechaza participar & 9,8 & 4,9 & 1,94 \\
9. El alumno toma un rol propio del docente & 0,5 & $5,8^{*}$ & $-2,99$ \\
\hline * Difercias estísicam
\end{tabular}

* Diferencias estadísticamente significativas; Prueba Z de proporciones: Z=1,96; a: 0,05

Fuente: elaboración propia.

Se observa a primera vista que las asambleas son espacios que propician que niños de 4 y 5 años repitan, elaboren y reestructuren información presente en el discurso compartido. En este sentido, se evidencia que la secuencia de asambleas ha contribuido, tanto en 4 como en 5 años, a un progresivo aumento de la reestructuración de información, esto es, al replanteamiento de la información dada, muy ligado al avance del conocimiento. El hecho de que en la asamblea 3, si bien con valores bajos, aparezcan intervenciones dirigidas a hacer preguntas en ambas aulas, refuerza este planteamiento.

En la Asamblea 1 la participación de los dos grupos muestra más similitudes que diferencias; en ambas aulas elaboran, reestructuran, dan información y, en menor medida, repiten la información previamente dada. La diferencia estriba en que en el grupo de 5 años se elabora con menos frecuencia que en 4 años. Resulta interesante que hay poca repetición literal de lo dicho previamente, cuando eso es bastante frecuente a estas edades (Monteira y Jiménez-Aleixandre, 2019).

En la Asamblea 2 la participación de los grupos difiere más. En 4 años las intervenciones que se limitan a repetir superan a las del aula de 5 años, y, aunque en 4 años hay elaboración de información, no llega a los valores de la clase de 5 años. Además, en esta aula los niños dan más cantidad de información respecto a los de 4 años. En ambas aulas, sin embargo, se reestructura la información con una alta frecuencia. Hay otra diferencia significativa y es que, en la clase de 5 años, si bien con un valor muy bajo, hay intervenciones en las que niños adquieren rol docente (demandan información, etc.) lo que indica un alto grado de implicación en el discurso compartido.

La participación del alumnado coincide en las dos aulas en la Asamblea 3 en la alta frecuencia de las intervenciones dirigidas a elaborar y reestructurar información dada. Se diferencian por la mayor frecuencia de repetición de información en 4 años y la mayor frecuencia de casos en que el alumnado asume rol del docente en 5 años. 


\section{3 ¿Qué esquemas de conocimiento afloran en las sesiones de asamblea?}

En relación con el plano conceptual, o patrón temático según Lemke (1997), en un estudio anterior mostramos los esquemas que afloran en cada una de las asambleas analizadas en el grupo de 5 años. Como allí se expone (Aragón et al., 2021), en la asamblea inicial se identificaron un total de 7 esquemas vinculados con modelos iniciales similares a los mostrados en el aula de 4 años: el modelo de huerto y el modelo de plantas como seres vivos. En la asamblea 2 y 3 , se identificaron un total de 22 y 27 esquemas, respectivamente.

En el caso del aula de 4 años, se han identificado 30 esquemas de conocimiento científico a lo largo de las tres asambleas: 10 en la primera asamblea, 17 en la segunda y 3 en la tercera. Algunos de estos esquemas fueron aportados por la propia docente, como es el caso del esquema: "las plantas hay que cuidarlas" en la secuencia 1 de la asamblea 2, o "en el huerto hay bichitos", en la secuencia 1 de la asamblea 3. Por otro lado, los esquemas mentales del alumnado de 4 años giran principalmente en torno a cuatro modelos precursores explicativos: el modelo de las plantas como seres vivos, el modelo del ciclo de vida de las plantas, el modelo de huerto y el modelo de huerto ecológico. Estos se muestran separados por asambleas y secuencias según orden de aparición (Tabla 6).

Tabla 6. Esquemas y modelos iniciales explicativos emergentes identificados en la asamblea 1,2 y 3 en el aula de 4 años.

\begin{tabular}{|c|c|c|}
\hline \multicolumn{3}{|c|}{ Asamblea 1} \\
\hline Secuencia & Esquemas de conocimiento & $\begin{array}{l}\text { Modelos mentales pre- } \\
\text { cursores }\end{array}$ \\
\hline \multirow[t]{6}{*}{ g1)¿Qué es un huerto? } & 1) Huerto como lugar donde nace & Modelo de huerto \\
\hline & comida & \multirow{5}{*}{$\begin{array}{l}\text { Modelo de ciclo de } \\
\text { vida de las plantas } \\
\text { Modelo de huerto } \\
\text { Modelo de las plantas } \\
\text { como seres vivos }\end{array}$} \\
\hline & 3) Las plantas salen de una maceta & \\
\hline & $\begin{array}{l}\text { 4) El huerto es un lugar sano donde } \\
\text { hay frutas y verduras }\end{array}$ & \\
\hline & $\begin{array}{l}\text { 5) El huerto como lugar donde se } \\
\text { plantan flores }\end{array}$ & \\
\hline & $\begin{array}{l}\text { 6) Las plantas necesitan agua para } \\
\text { crecer }\end{array}$ & \\
\hline \multirow{3}{*}{$\begin{array}{l}\text { 2)¿Qué hay en un } \\
\text { huerto?/¿Qué necesitan las } \\
\text { plantas para crecer? }\end{array}$} & 7) Las plantas necesitan tierra & \multirow{3}{*}{$\begin{array}{l}\text { Modelo del ciclo de } \\
\text { vida de las plantas }\end{array}$} \\
\hline & $\begin{array}{l}\text { 8)Las plantas necesitan jardín/campo } \\
\text { para crecer }\end{array}$ & \\
\hline & $\begin{array}{l}\text { 9) las plantas pueden nacer en un } \\
\text { vaso de yogurt }\end{array}$ & \\
\hline $\begin{array}{l}\text { 3)¿Qué se planta en un } \\
\text { huerto? }\end{array}$ & 10) Las plantas tienen raíces & $\begin{array}{l}\text { Modelo las plantas } \\
\text { como seres vivos }\end{array}$ \\
\hline
\end{tabular}




\section{Asamblea 2}

\begin{tabular}{|c|c|c|}
\hline Secuencia & Esquemas de conocimiento & $\begin{array}{l}\text { Modelos mentales } \\
\text { precursores }\end{array}$ \\
\hline \multirow[t]{3}{*}{$\begin{array}{l}\text { 1)¿Qué hace falta en un } \\
\text { huerto? }\end{array}$} & $\begin{array}{l}\text { 1) Las plantas necesitan algodón } \\
\text { para crecer }\end{array}$ & $\begin{array}{l}\text { Modelo de las plantas } \\
\text { como seres vivos }\end{array}$ \\
\hline & 2) las plantas hay que cuidarlas & Modelo de ciclo de \\
\hline & 3) las plantas necesitan luz /sol & vida de las plantas \\
\hline \multirow{11}{*}{$\begin{array}{l}\text { 2) Lista de cosas que hay en } \\
\text { un huerto }\end{array}$} & 4) El huerto necesita agua & Modelo de huerto \\
\hline & 5) El huerto necesita sol & \\
\hline & 6) En el huerto hay semillas & \\
\hline & 7) El huerto necesita tierra & \\
\hline & 8) En el huerto hay quesos & \\
\hline & 9) El queso sale de una fábrica & \\
\hline & 10) EI huerto necesita viento & \\
\hline & 11) En el huerto no hay garbanzos & \\
\hline & $\begin{array}{l}\text { 12) Los garbanzos se plantan en el } \\
\text { huerto }\end{array}$ & \\
\hline & 13) En el huerto hay chocolate & \\
\hline & 14) En el huerto hay pescado. & \\
\hline \multirow[t]{3}{*}{$\begin{array}{l}\text { 3) Cosas que hacen falta en } \\
\text { el huerto/acciones }\end{array}$} & $\begin{array}{l}\text { 15) El huerto como lugar donde hay } \\
\text { herramientas y se hacen acciones }\end{array}$ & $\begin{array}{l}\text { Modelo de huerto } \\
\text { Modelo de huerto }\end{array}$ \\
\hline & 16) En el huerto hay bichitos & ecológico \\
\hline & 17) El huerto como lugar ecológico & \\
\hline \multicolumn{3}{|c|}{ Asamblea 3} \\
\hline Secuencia & Esquemas de conocimiento & $\begin{array}{l}\text { Modelos mentales } \\
\text { precursores }\end{array}$ \\
\hline 1) Factor luz/sol & $\begin{array}{l}\text { 1) La luz es necesaria para germinar } \\
\text { la semilla }\end{array}$ & $\begin{array}{l}\text { Modelo de ciclo de } \\
\text { vida de las plantas }\end{array}$ \\
\hline \multirow[t]{2}{*}{ 2) Factor profundidad } & $\begin{array}{l}\text { 2) Las semillas que están arriba salen } \\
\text { antes que las de abajo }\end{array}$ & $\begin{array}{l}\text { Modelo de las plantas } \\
\text { como seres vivos }\end{array}$ \\
\hline & $\begin{array}{l}\text { 3) Las semillas que están más abajo } \\
\text { también salen. }\end{array}$ & \\
\hline
\end{tabular}

Fuente: elaboración propia.

Desde una mirada adulta puede parecer que algunos de estos esquemas no guardan a priori relación con el huerto; sin embargo, consideramos que poseen un valor significativo para generar movimiento en el discurso científico compartido. Es el caso de esquemas como "En el huerto hay quesos", o "En el huerto hay chocolate". Un ejemplo de ello se evidencia en la siguiente conversación (N: Niño/a, M: Docente).

$\mathrm{N}$ : chocolate.

M: chocolate, ¿el chocolate también se siembra?

N: no.

N: sí. 
M: bueno habéis dicho chocolate, yo lo pongo y ahora me decís. A ver, C. ¿qué dices tú del chocolate?

$\mathrm{N}$ : que no porque un chocolate no es natural.

M: ¿Qué un chocolate no es natural? ¿eso qué quiere decir?

$\mathrm{N}$ : que se derrite con el sol

M: ah, que se derretiría con el sol el chocolate ¿no?

$N$ : sí.

Comparando las dos aulas, se observa que en ambas los esquemas explicitados se relacionan con los modelos iniciales del ciclo de vida de una planta y el modelo de plantas como seres vivos, pero con algunas diferencias: el alumnado de 5 años parece haber asimilado mejor estos esquemas, principalmente, con relación al modelo de las plantas como seres vivos. En 4 años se explicitan menos esquemas relacionados con las necesidades de las plantas, y parece haber dificultades para vincular estos esquemas con los experimentos que se mencionan en la asamblea 3, a diferencia del grupo de 5 años, que busca explicaciones en función de los esquemas aportados en las diversas asambleas. No obstante, el alumnado de 4 años ya maneja ideas muy interesantes sobre el huerto, como "lugar donde nace comida", o la idea de ecológico "como lugar sano donde hay frutas y verduras" o "lugar donde se cuidan las plantas".

\section{Discusión y conclusiones}

Los resultados aportados evidencian que el huerto ecológico escolar es un contexto de aprendizaje adecuado para promover la competencia científica en edades tempranas (Eugenio-Gonzalbo et al., 2020; Fernández-Arroyo et al., 2013). Las asambleas suponen espacios propicios para generar discurso científico tanto en el aula de 4 años como en 5 años pues propician el recurso al habla exploratoria colectiva (Mercer, 2017). La intervención docente y la participación infantil se adaptan a la progresiva complejidad de las asambleas programadas en la secuencia didáctica, si bien se observan diferencias significativas entre los grupos: en cuanto a la participación, el grupo de 4 años requiere un mayor apoyo docente para promover las intervenciones infantiles, pero los logros en la participación-la alta presencia en las tres asambleas de repetición, elaboración y reestructuración de información, y el progresivo aumento de la reestructuración a lo largo de la secuencia- dan cuenta del éxito en la consecución de un avance en el discurso científico compartido. En el caso del aula de 5 años, se observa un discurso más retador por parte del docente, que responde también a una adaptación a las posibilidades infantiles - los datos muestran que los niños de 5 años recurren en menor medida a la repetición de información para, en cambio, elaborar o reestructurar con más frecuencia-. Con este estudio, por tanto, mostramos planteamientos didácticos que integran la educación científica y la socialización (Cantó et al., 2016) y subrayan la importancia de trabajar la expresión y comunicación de ideas en torno a la ciencia (Sardá y Sanmartí, 2000). También, señalamos la relevancia de familiarizar desde edades tempranas con un discurso que requiere de confrontación y validación de hechos para ser aceptado (Monteira y Jiménez Aleixandre, 2016; 2019), mostrando además su concreción en 4 y 5 años. 
Por otro lado, los avances en la construcción del discurso se traducen en el plano conceptual en la explicitación de esquemas mentales diversos en torno a los fenómenos experimentados en el huerto ecológico. Estos esquemas conectan con modelos iniciales precursores de gran valor para avanzar a los modelos más complejos en cursos sucesivos. Este conocimiento emergente está presente desde los 4 años, aunque con menor complejidad que a los 5 años (Aragón et al., 2021). Es relevante también cómo afloran ideas, quizás más alejadas del conocimiento científico escolar deseable, o carentes de sentido desde la perspectiva del docente, pero igualmente valiosas puesto que desde ellas es posible hacer crecer el conocimiento compartido en la etapa, a través de estrategias de intervención docente (apoyar intervenciones, retar planteamientos) que permitan al alumnado no solo aportar información, sino elaborarla y reestructurarla, en consonancia con el desarrollo del contenido conceptual asociado a las experiencias sobre las que se conversa.

Otros estudios han puesto ya de manifiesto el interés de promover la competencia científica desde cursos anteriores (Cantó et al., 2016; García-Carmona et al., 2014), por lo que consideramos necesario continuar indagando en propuestas didácticas que afecten al $2^{\circ}$ ciclo completo de El, y que contribuyan a visibilizar tanto las capacidades infantiles como las estrategias docentes que promueven la competencia científica en edades tempranas, además de visibilizar las posibilidades del huerto ecológico escolar como contexto idóneo para desarrollar aprendizajes propios de la ciencia a través de enfoques diversos e investigativos (Aragón y Morilla, 2021).

\section{Referencias}

Alcántara, J., Torres-Porras, J., Mora, M., Rubio, S.J., Arrebola, J.C,. y Rodríguez, L. (2019). ¿Son los huertos escolares en educación infantil una realidad o una innovación educativa? Estudio de centros escolares de la ciudad de Córdoba (España) y propuestas de cambio desde la Universidad. Didáctica de las Ciencias Experimentales y Sociales, 36, 79-96. https:// doi.org/10.7203/DCES.36.12535.

Aragón, L. y Morilla, B. (2021). El uso del huerto escolar en los centros de educación infantil de la Campiña Morón-Marchena (Sevilla). Una mirada desde la competencia científica en educación infantil. Campo Abierto, 40(2), 187-206.

Aragón, L. y Sánchez, S. (aceptado). Intervención docente en el discurso científico en el aula de infantil en torno al huerto ecológico escolar. En $11^{\circ}$ Congreso Internacional sobre Investigación en la Didáctica de las Ciencias.

Aragón, L., Sánchez, S. y Enríquez, J.M. (2021). El discurso científico en la etapa de infantil en el contexto del huerto ecológico escolar. Revista Eureka sobre Enseñanza y Divulgación de las Ciencias, 18(1), 1103. https://doi.org/10.25267/Rev_Eureka_ensen_divulg_cienc.2021. V18.i1.1103

Armienta, D.E., Keck, C., Ferguson, B. y Saldívar, A. (2019). Huertos escolares como espacios para el cultivo de relaciones. Innovación Educativa, 161-178, 19(80), Recuperado de https:// bit.ly/38wULBP

Baker, M.J., Andriessen, J. y Schwarz, B.B. (2019). Collaborative argumentation-based learning. En Neil Mercer, Rupert Wegerif and Louis Major (Eds.) The Routledge International Handbook of Research on Dialogic Education. (76-88). Abingdon: Routledge. 
Canedo-Ibarra, S.P., Castelló-Escandell J., García-Wehrle P., Gómez-Galindo A. y Morales-BlakeA.R. (2012). Cambio conceptual y construcción de modelos científicos precursores en educación infantil. Revista Mexicana de Investigación Educativa, 17(54), 691-727.

Cantó, J., de Pro, A. y Solbes, J. (2016). ¿Qué ciencias se enseñan y cómo se hace en las aulas de educación infantil? La visión de los maestros en formación inicial. Enseñanza de las Ciencias, 34(3), 25-50.

Carballido, J.L, Morón-Monge, H. y Daza, Ma . P. (2021). El huerto escolar desde un enfoque indagativo: investigando las lombrices. Investigación en la Escuela, 103, 75-93. https:// doi.org /10.12795/IE.2021.i103.06

De la Blanca, S., Hidalgo, J. y Burgos, C. (2013). Escuela infantil y ciencia: la indagación científica para entender la realidad circundante. En X Congreso Internacional sobre Investigación en Didáctica de las Ciencias, 979-983. Recuperado de https://bit.ly/2Wmgmuh

Díez, C. (1998). La oreja verde de la escuela: trabajo por proyectos y vida cotidiana en la escuela infantil. Madrid: Eds. de la Torre - Proyecto Didáctico Quirón.

Fernández-Arroyo, J., Puig, M. y Rodríguez-Marín, F. (2013). El uso del huerto escolar en la formación del profesorado de magisterio a través de la práctica educativa. In M. A., BallesteriosMoscopios y F., Ries (Eds), International Conference Re-conceptualizing the professional identity of the European teacher. Sharing Experiences (pp.699-716). Copiarte. http://hdl. handle.net/11441/56705

Eugenio-Gozalbo, M., Aragón, L. \& Ortega-Cubero, I. (2020). Gardens as science learning contexts across educational stages: Learning assessment based on students' graphic representations. Frontiers in Psychology, 11, 2226. https://doi.org/10.3389/fpsyg.2020.02226

Eugenio, M. y Aragón, L. (2016). Experiencias en torno al huerto ecológico como recurso didáctico y contexto educativo en la formación inicial de maestros/as. Revista Eureka sobre Enseñanza y Divulgación de las Ciencias, 13(3), 667-679. Recuperado de https://bit.ly/2XS100H

García. J.E. (2003). Investigando el ecosistema. Investigación en la Escuela, 51, 83-100.

García-Carmona, A., Criado, A.M. y Cañal, P. (2014). Alfabetización científica en la etapa 3-6 años: un análisis de la regulación estatal de enseñanzas mínimas. Enseñanza de las Ciencias, 32(2), 131-149. https://doi.org/10.5565/rev/ensciencias.817

Garzón, A. y Martínez, A. (2017). Reflexiones sobre la alfabetización científica en la educación infantil. Espiral. Cuadernos del Profesorado, 10(20), 28-39. Disponible en http://espiral. cepcuevasolula.es/

Germinando (2018). Germinando espacio agroecológico. Recuperado de https://bit.ly/3kyN2c1

Izquierdo, M. y Sanmartí, N. (1999). Enseñar a leer y escribir textos de Ciencias de la Naturaleza. En J. Jorba, I. Gómez, A. Prat (Eds.), Hablar y escribir para aprender. (pp. 181-200) Síntesis, Madrid.

Latorre, A., Del Rincón, D. y Arnal, J. (2003). Bases metodológicas de la investigación educativa. Barcelona: Ediciones Experiencia.

Lefstein, A. y Snell, J. (2011). Classroom discourse: The promise and complexity of dialogic practice. En S. Ellis y E. McCartney (Eds.), Applied Linguistics and Primary School Teaching (165185). Cambridge: Cambridge University Press.

Lefstein, A. y Snell, J. (2019). Linguistic ethnographic analysis of classroom dialogue. En En Neil Mercer, Rupert Wegerif and Louis Major (Eds.) The Routledge International Handbook of Research on Dialogic Education. (63-75). Abingdon: Routledge.

Lemke, J. (1997). Aprender a hablar ciencia: lenguaje, aprendizaje y valores. Barcelona: Paidós.

Marques, T.J. y Cuéllar, M. (2021). Los huertos escolares y su potencial como innovación educativa. Enseñanza de las Ciencias, 39(2), 163-180. https://doi.org/10.5565/rev/ensciencias.2886

Mazas, B., Gil-Quílez, M.J., Martínez-Peña B., Hervás, A. y Muñoz, A. (2018). Los niños de infantil piensan, actúan hablan sobre el comportamiento del aire y del agua. Enseñanza de las Ciencias, 36(1), 163-180. 
Mercer, N. (2017). La importancia educativa del habla. En N. Mercer, L. Hargreaves y R. García. Aprendizaje e Interacciones en el Aula. (15-35). Barcelona: Hipatia.

Mercer, N., Wegerif, R. y Major, L. (2019). The Routledge International Handbook of Research on Dialogic Education. Abingdon: Routledge.

Mérida, R. Torres-Porras, J. y Alcántara, J. (2017). Didáctica de las ciencias experimentales en educación infantil. España: Editorial Síntesis.

Mérida, R., González, E. y Olivares, M. (2016). Estrategias y modalidades de argumentación oral en las asambleas de Educación Infantil. Un estudio multicaso. Revista Complutense de Educación, 28(2), 445-462. https://doi.org/10.5209/rev_RCED.2017.v28.n2.49439

Monteira, S. F. y Jiménez-Aleixandre, M. P. (2016). The practice of using evidence in kindergarten: The role of purposeful observation. Journal of Research in Science Teaching, 53(8), 1232-1258.

Monteira, S. F. y Jiménez-Aleixandre, M. P. (2019). ¿Cómo llega el agua a las nubes? Construcción de explicaciones sobre cambios de estado en educación infantil. Revista Eureka sobre Enseñanza y Divulgación de las Ciencias, 16(2), 2101. https://doi.org/10.25267/Rev_ eureka_ensen_divulg_cienc.2019.v16i2.2101.

Mortimer, E. F. y Scott, P. H. (2003). Meaning making in secondary science classrooms. Maidenhead: Open University Press

Pozuelos, F.J. (2007). Trabajo por proyectos: descripción, investigación yexperiencias. Morón: Ediciones MCEP: Cooperación Educativa.

Real Decreto 1630/2006, de 29 de diciembre, por el que se establecen las enseñanzas mínimas del segundo ciclo de Educación infantil. Disponible en https://bit.ly/3BlqguX

Rodríguez-Marín, F., Fernández-Arroyo, J. y García-Díaz, J.E. (2015). El huerto escolar ecológico como herramienta para la educación en y para el decrecimiento. Investigación en la Escuela, 86, 35-48.

Rodríguez-Marín, F., Portillo M. A. y Puig M. (2021). El Huerto Escolar como recurso para iniciar la Alfabetización Ambiental en Educación Infantil. Revista Eureka sobre Enseñanza y Divulgación de las Ciencias 18 (2), 2501. https://doi.org/10.25267/Rev_Eureka_ensen_ divulg_cienc.2021.v18.i2.2501

Sáez, M. J., Lucha, P., Claver, A. M., Arasanz Broto, A. y Iráizoz Esteban, R. (2017). Del dicho al hecho en una propuesta sobre ecosistemas contextualizada en el huerto escolar. Ápice. Revista de Educación Científica, 1(2), 47-57. https://doi.org/10.17979/arec.2017.1.2.3085

Sánchez, S. y González, C. (2016). La asamblea de clase en Educación Infantil: un espacio para crecer como grupo. Revista Iberoamericana de Educación, 71, 133-150. https://doi. org/10.35362/rie7108

Sánchez, S. (2016). Hablar para comunicarse. En Fons-Esteve, Montserrat; Palou Sangrà, Juli (Coords). Didáctica de la lengua y la literatura en Educación Infantil. (pp.165-178). Madrid: Síntesis.

Sanmartí, N. (2002). Un reto: mejorar la enseñanza de las ciencias en Las Ciencias en la Escuela. Barcelona: Graó.

Sardá, A. y Sanmartí, N. (2000). Ensenyar a argumentar científicament: un repte de les clases de ciències. Enseñanza de las Ciencias, 18(3), 405-422.

Stake, R. E. (2005). Investigación con estudio de casos. Madrid: Morata

Warwick, P. y Cook, V. (2019). Classroom dialogue. En En Neil Mercer, Rupert Wegerif and Louis Major (Eds.) The Routledge International Handbook of Research on Dialogic Education. (121-124). Abingdon: Routledge.

Wells, G. (1987). Apprenticeship in literacy. Interchange, 18(1-2), 109-123.

Wells, G. (2001). Indagación dialógica. Barcelona: Paidós.

Yin, R. K. (2009). Case study research: Design and methods (4th Ed.). Thousand Oaks, CA: Sage.

\section{Cómo citar en APA:}

Aragón, L., Sánchez,S. y García, V. (2021). Sostener el discurso científico en aulas de 4 y 5 años: análisis de las intervenciones de docentes y alumnado y del conocimiento emergente en torno al huerto ecológico escolar. Revista Iberoamericana de Educación, 87(1), 135-153. https:// doi.org/10.35362/rie8714606 